\title{
Application of Fuzzy Comprehensive Evaluation in Cognitive Networks for Optimal Network Selection
}

\author{
Xiaoming Chen*, Huiqiang Wang, Hongwu Lv, Amjad Ali and Zhendong Wang \\ College of Computer Science and Technology, Harbin Engineering University, Harbin 150001, P. R. China
}

Received: 12 Jul. 2013, Revised: 25 Nov. 2013, Accepted: 26 Nov. 2013

Published online: 1 Jan. 2014

\begin{abstract}
Network selection mechanisms play a vital role in ensuring quality of service (QoS)in the cognitive networks environment. In this paper we develop a network selection scheme for an integrated UMTS/WLAN system to guarantee mobile users being always best connected. We focus on the optimization of resource utilization, while ensuring acceptable QoS provision to the end users. The proposed scheme comprises three parts. First one is to detect the availability of access networks by following cognitive network architecture under the cross-layer design paradigm. While the second is to apply fuzzy comprehensive evaluation to decide the relative weights of evaluative criteria set according to network condition and service applications. Thirdly, a novel approach using quantum genetic algorithm to adjust the weights of fuzzy comprehensive evaluation is adopted. Extensive computer based simulation experiments were carried out. Simulation results show that this method gives better performance in throughput, delay and average packet loss, hence proves that this method is superior to the traditional methods of link capacity and network capacity.
\end{abstract}

Keywords: Fuzzy Comprehensive Evaluation, Cognitive Network, Quantum Genetic Algorithm

\section{Introduction}

In recent years, with the development of wireless communication systems, different wireless networks make it attractive to integrate a wide range of access technology standards [1,2], including WLANs and UMTS. To select an optimal access network based on different user requirements, and at the same time ensure the efficient allocation of network resources and improve the overall network performance, is a critical issue in heterogeneous wireless network environment $[3,4]$.

To resolve this issue efficiently, its paramount to have a comprehensive knowledge of the state of the network environment and make sure decisions are made intelligently. Cross-layer design $[5,6]$ is crucial in obtaining a comprehensive view of the information exchange between various layers. Cognitive network techniques in recent years have been seen by researchers to have the potential to improve the overall network performance.

To resolve this problem, how to get the comprehensive perception of network information and intelligent decision is crucial. At present cognitive networks technology has been considered to be realized the key of optimizing the network global performance [7].
The idea of cognitive networks was first introduced by Virginia Tech University and is defined [8] as "a network with a cognitive process that can perceive current network conditions, and then plan, decide and act on those conditions". A cognitive network keeps track of various network parameters and adapts itself to achieve better performance. The network can learn from these adaptations and use them to make future decisions, all while taking into account the end-to-end goals. Therefore, cross-layer method has been used in conjuncture with cognitive network techniques for selecting an appropriate access network. Cross-layer architectures provide a holistic view of the cross-layer design model [9], focusing on how to interface or integrate layers from across the network protocol stack.

A number of methods have been proposed for network access selection in the recent past that has added considerable improvements. Most previous solutions dealing with this problem has focused only on a specific layer. The most notable contributions are as follows. The work in [10] has proposed a probability model based on the adjacent channel classification according to network spectrum utilization. In this study, the secondary users accessing spectrum resources of the primary users do not

\footnotetext{
*Corresponding author e-mail: chenxiaoming @ hrbeu.edu.cn
} 
affect the performance of primary users and also ensures that spectrum resources are utilized to the maximum possible. The study carried out in [11], proposed an access selection method based on RFB fuzzy neural network focused on multi-access link-layer load balancing and adaptive modulation mechanism. The authors in $[12,13]$ proposed a network access selection algorithm based on Markov decision process, considering the bandwidth at the network layer and the connection delay. The authors in $[14,15]$ have proposed an access network selection algorithm based on user services at the application layer. This paper uses mix-strategy Nash equilibrium used in games to optimize network performance. Our approach is different since our goal is to achieve a desired throughput, average packet delay and packet loss by selecting an access network adaptively, on best performance basis. Our solution is two pronged, cognitive network as an access network and also using a cross-layer design.

The rest of this paper is organized as follows. In section two we describe our proposed architecture for the cognitive process based on MDE cycle. A novel method for network access is developed in Section 3. Results attained by performing the designed simulation experiments are reported in Section 4. Finally, we conclude this article and present future possibilities in this area in Section 5.

\section{The Proposed Optimal Network Selection Architecture}

There are two steps to make a decision to select the best network. The firsts step is collecting all necessary information that may affect selection. The second step is under a certain method among these demands.

\subsection{Cognitive Network Architecture}

Here we present the cognitive network architecture to achieve optimum network parameters. Cognitive cycle is crucial in realizing an adaptive access network selection. This process contains three parts (MDE): Monitor, Decide and Execute. Each mobile terminal includes a MDE cycle. The proposed Architecture based on cross-layer design and the specific implementation of decision-making are illustrated in Fig. 1. The model mainly consists of two parts: cognitive cycle and a network protocol stack that interactively communicate through a cross-layer bus.

Cognitive Cycle: The Monitor component (MC) provides important information about the characteristics or behavior of the protocols and the status of the network. After filtering out the required information from the original set of information, integration and normalization process, the information is submitted to the decision component (DC). DC evaluates the performance of the current access network according to the cognitive repository, and based on the existing strategy, generate event set and assign tasks and resources. Execution component (EC) chooses an optimal/subprime access network based on the selected strategy. The cognitive repository is updated after the implementation of the strategy. On the basis of MDE cognitive process, the DC adaptively selects an access network that offers best performance to the end user.

Network Protocol Stack: provide normal layering support when it is necessary. This allows full compatibility with standards and maintains the benefits of a modular architecture, as it does not modify each layer's core functionality. Each layer in the existing protocol stack will have a corresponding connector between the layer and cross-layer bus. It also provides the different layers context information to cognitive layer through the cross-layer bus.

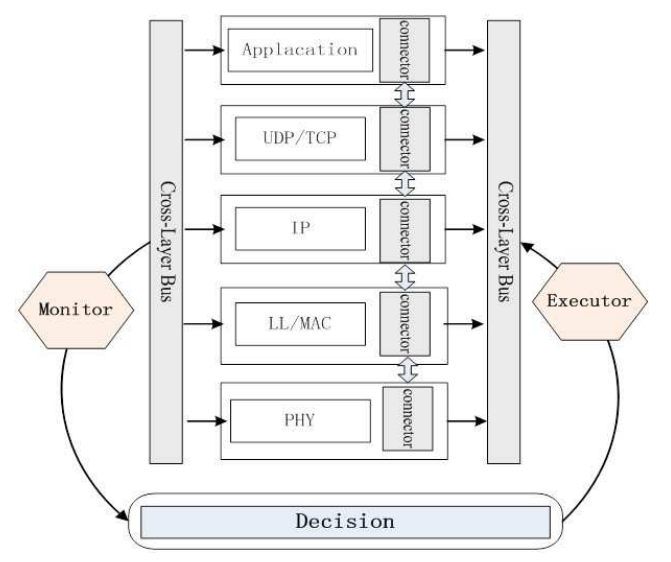

Fig. 1: The Cognitive Network Architecture Based on MDE

Cross-layer Bus and Connector: In order to simplify the complexity of the cross layer design, each layer needs to understand the behavior of the other layers. Each level contains special connectors, and interacts through crosslayer bus with the cognitive process.

\subsection{Decision Model Operation}

Fig. 2 shows that in reality, there are two control loops in the Decision Component. The desired state of the application is predefined in cognitive repository (CR), and based on user goals. The bottom (maintenance) control loop is used when $\operatorname{Net}_{k}(k=1,2, \ldots, N)$ meets the QoS of user. The top (adjustment) control loop is used when the desired user goals cannot be me. The use of two different control loops, one for maintenance operations and one for reselection operations is fundamental to overcoming the limitations of using a single static control loop. 


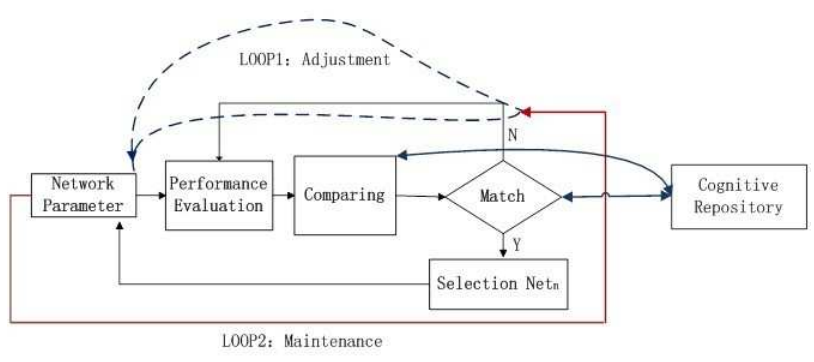

Fig. 2: The Function of Decision Component

In this process, the parameters are first collected by MC. After filtering the original information, integration and normalization process, the information is submitted to the DC. The fuzzy comprehensive evaluation method was used to achieve the optimal solution. The performance of $\mathrm{Net}_{k}$ compared with that of the already stored value in cognitive repository. If the two matched, then $\mathrm{Net}_{k}$ is selected, otherwise the access network is adjusted according to the optimal solution configuration in the cognitive repository. The process of implementation is a layer by layer configuration process in the descending order, but also an optimization process from bottom layer to the topmost layer in ascending order.

\section{Problem Description and Solution}

Most networks are very complex and dynamic in nature and a number of parameters may change without any predictable pattern. This often results in uncertain and variable performance and makes it very difficult to set a precise performance level for access networks. Fuzzy comprehensive evaluation method [16] can be used to achieve much better results in such circumstance.

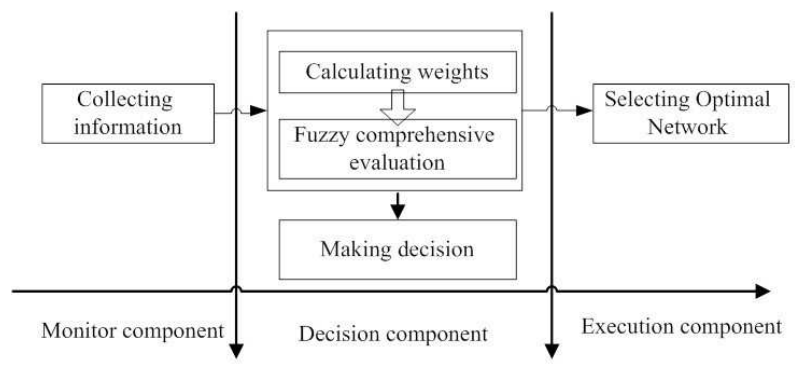

Fig. 3: The Function of Decision Component

The whole network selection model is shown in Fig. 3 The network selection mechanism is usually divided into three steps: collecting information, making decision and executing the best selection. Here we consider integrated UMTS and WLAN as cognitive networks environment.

\subsection{Fuzzy comprehensive evaluation for access selection algorithm}

DC of the MDE Cycle is used to realize the fuzzy comprehensive evaluation process. Fuzzy comprehensive evaluation method is useful in network quality of service assessment, which adopts the principle of fuzzy relationship synthesis. It comprehensively judges the membership grade status of various factors to be judged from many factors. The basic steps of fuzzy comprehensive evaluation method are as follows.

Step1: Establish the comprehensive evaluation factor set. This factor set is composed of the elements such as reliability, packet loss, delay, jitter and throughput. It is expressed by $U$, That is

$$
U=u_{1}, u_{2}, \ldots, u_{n}
$$

Where $u_{i}(1 \leq i \leq n)$ represents the $i$ evaluation factor.

Step2: Set up comprehensive evaluation set. Evaluation set is composed of various kinds of results generated after the evaluation process to judge the access network for best performance for various scenarios. Evaluator making for the judged access network Net $_{k}$, expressed by $V$, that is

$$
V=v_{1}, v_{2}, \ldots, v_{m}
$$

Among the elements, $v_{j}(1 \leq j \leq m)$ represents the $j$ evaluation result, five assessment grades can be determined. The set can be expressed as:

$$
\begin{aligned}
V & =\left\{v_{1}, v_{2}, \ldots, v_{5}\right\} \\
& =\{\text { seamless, excellent }, \text { better }, \text { good }, \text { bad }\}
\end{aligned}
$$

Step3: Carry on fuzzy evaluation for single factor and obtain the evaluation matrix. According to step 2, establish a single factor evaluation matrix $R$. The relationship between the factor set and the evaluation set use fuzzy matrix representation $R$ by the membership function.

$$
\begin{gathered}
R=\left(\begin{array}{c}
R_{1} \\
R_{2} \\
\vdots \\
R_{m}
\end{array}\right)=\left(\begin{array}{cccc}
r_{11} & r_{12} & \cdots & r_{1 n} \\
r_{21} & r_{22} & \cdots & r_{2 n} \\
\vdots & \vdots & \vdots & \vdots \\
r_{n 1} & r_{n 2} & \cdots & r_{n m}
\end{array}\right), \\
0 \leq r_{i j} \leq 1,0 \leq i \leq n, 0 \leq j \leq m .
\end{gathered}
$$

Where $R$ denotes a $m \times n$ dimensional evaluation matrix, $i$ represents the number of the influence factors and $j$ is the level in evaluation set $(V) . r_{i j}$ is membership of ith influence factor, which stand for a special network layer performance parameter variable. Evaluation space represents the triplet, expressed as $(U, V, R)$. 
Step4: Establish weight vectors for various parameters. Assuming that a set of weight factors $(W)$ is used to account for relative importance of all influence factors, $W$ can be described as follows:

$$
W=\left(w_{1}, w_{2}, \ldots, w_{n}\right), \quad \sum_{i=1}^{n}=1, w_{i}>0,1 \leq i \leq n .
$$

In this paper we have used quantum genetic algorithm to estimate the weight parameter.

Step5: Set up comprehensive evaluation model. The results of the comprehensive evaluation model can ultimately be calculated by the following equation:

$$
\begin{aligned}
B & =W \circ R \\
& =\left(w_{1}, w_{2}, \ldots, w_{i}\right) \circ\left(\sum_{i=1}^{4} w_{i} \times r_{i j}\right) \\
& =\left(b_{1}, b_{2}, \ldots, b_{j}\right)
\end{aligned}
$$

The past user experiences with different access networks that is stored in the cognitive repository is obtained from the cognitive repository and compared with the current status of the access network. Then quantum genetic algorithm ( $Q G A)$ was applied to calculate the value of weight vectors $(W)$ with individual networks with separate AP's $\mathrm{Net}_{1}, \mathrm{Net}_{2}, \ldots, \mathrm{Net}_{k}$. Where $W$ is the weight vectors associated with each application layer service which may vary from one application to another. For example in the case of VoIP low latency links are critical for its operation, for file transfer reliability is the key requirement and for multimedia contents such as video conferencing, bandwidth is the key requirement. This method uses the principle of maximum membership function to select the best possible network for the user.

\subsection{Optimization weights of network factors based on $Q G A$}

The ultimate goal of fuzzy comprehensive evaluation is to acquire the overall evaluation of the network performance. It is very important to get single network evaluation weight parameters. The process to determine weight factor is actually searching for a set of optimal values, and makes the result of fuzzy relationship equations achieve consistency during live network operation. Quantum genetic algorithms provides excellent optimization feature for optimizing weight vectors $(W)$ associated with different application layer services.

$Q G A$ [17] is a probabilistic algorithm that is similar to genetic algorithm $(G A)$. $Q G A$ maintains a population of qubits chromosomes. It is based on the concept and principles of quantum computing.

$Q G A$ maintains a population of Q-bit individuals, $Q(t)=\left\{q_{1}^{t}, q_{2}^{t}, \ldots, q_{n}^{t}\right\}$, at generation , where is the size of population, and is a Q-bit individual defined as

$$
q_{j}^{t}=\left[\begin{array}{c|c|c|c}
\alpha_{1}^{t} & \alpha_{2}^{t} & \ldots & \alpha_{m}^{t} \\
\beta_{1}^{t} & \beta_{2}^{t} & \ldots & \beta_{m}^{t}
\end{array}\right], 1 \leq j \leq m
$$

Where $m$ is the number of Q-bits in Eq. 7 Initialization $Q(t), \alpha_{i}$ and $\beta_{i}$ of all $q_{j}^{0}$ are initialized with $1 / \sqrt{2}$. QGA algorithms are used to determine the weights of each layer weight vectors. The procedure of QGA is described as follows.

According to the current optimal individual $P_{\text {currentbest }}(t)$ get $Q_{\text {guide }}$, then the next generation of quantum population can be represented as:

$$
\begin{array}{r}
Q_{\text {guide }}(t)=\alpha \cdot P_{\text {currentbest }}(t)+(1-a) \cdot\left[1-P_{\text {currentbest }}(t)\right] \\
Q(t+1)=Q_{\text {guide }}(t)+b . n o r m r n d(0,1)
\end{array}
$$

Where $\alpha$ the impact factor of is $P_{\text {currentbest }}(t), b$ is the variance of the randomness in quantum population. In order to obtain the higher accuracy for weight vector $W$, each vector element can be express as a 16-bit binary number which can be described as

$$
\begin{aligned}
& P=\left(a_{P H Y_{1}}, \ldots, a_{P H Y_{n}}, a_{M A C_{1}}, \ldots, a_{M A C_{n}}, \ldots\right) \\
& Q=\bar{P}=\left(\ldots, a_{M A C_{n}}, \ldots, a_{M A C_{1}}, a_{P H Y_{n}}, \ldots, a_{P H Y_{1}}\right)
\end{aligned}
$$

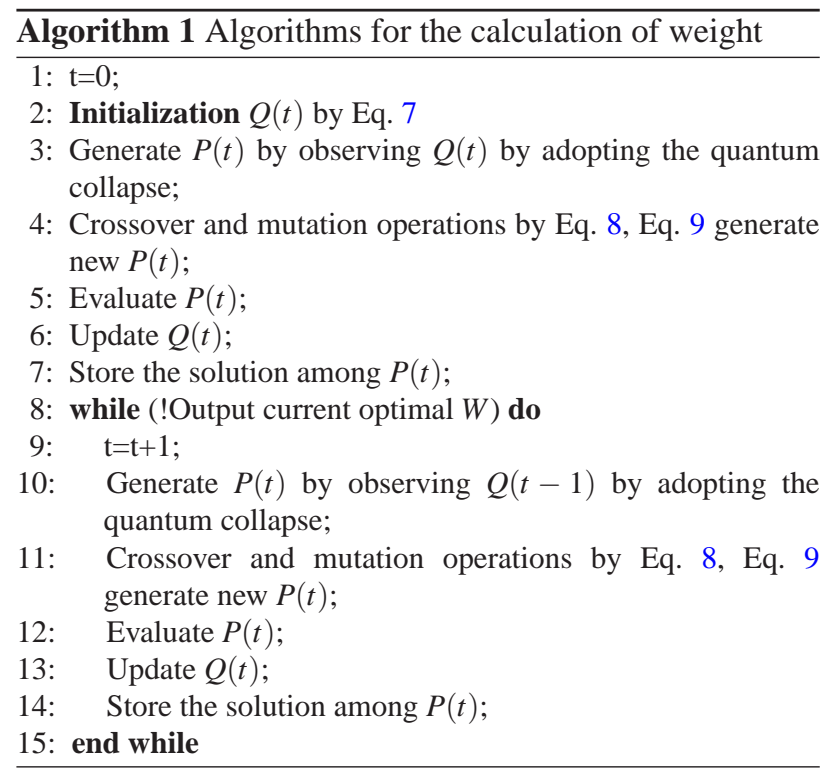

By using quantum swarm optimization algorithm, the most suitable weights in Step4 for fuzzy comprehensive evaluation are determined. The proposed algorithm, fuzzy comprehensive evolution based on QGA works as shown in algorithm 1.

\section{Simulation results}

We evaluate the proposed algorithm using NS2 which is a discrete event simulator [18]. We simulated a rectangular area of $600 \times 300$ with two access points were placed on either side of the center position, and $n$ randomly placed 
users. Each AP was connected to a fixed host with a dedicated symmetrical link whose bandwidth has a specified fixed value according to the considered scenario. For WLAN AP, we used $802.11 \mathrm{~g}$ with a rate adaptation scheme. For UTMS, we used a spreading factor of 8 in both downlink and uplink, which corresponds to a bit rate of $960 \mathrm{Kbps}$ in downlink and of $480 \mathrm{Kbps}$ in uplink. We considered several scenarios in order to evaluate different issues relevant to the network access selection problem. Each point on the simulation graph is an average of 60 simulation runs to make sure the results credible and more thorough.

\subsection{Comparing load balancing performance of the two access points with similar parameters}

The purpose of this scenario is to evaluate the load balancing capabilities of the different access schemes. We simulated a scenario in which the links connecting the two APs with fixed host have the same bandwidth (10Mbps). A maximum of 50 TCP users were added to the network gradually with time. The maximum simulation time is $600 \mathrm{~s}$. The result, reported in Fig. 4 for TCP users, show that in such a situation the link capacity method suffers severe performance degradation due to unbalanced load at the APs. Another scheme achieve a similar performance, with the Cognitive Network scheme achieving a slight throughput improvement over the others when there are enough users $(n>13)$ to provide sufficient statistical confidence for the performance estimation provided by the fuzzy cross-layer information fusion. As evident from Fig. 5, the Cognitive Network access method exhibit a smaller delay jitter, this is due to the fact that performance estimation provided by the cognitive repository.

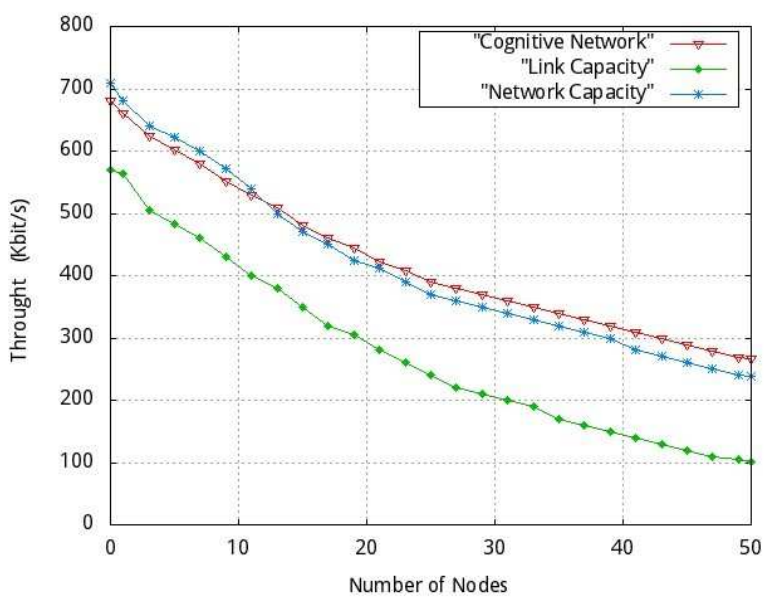

Fig. 4: Average throughput

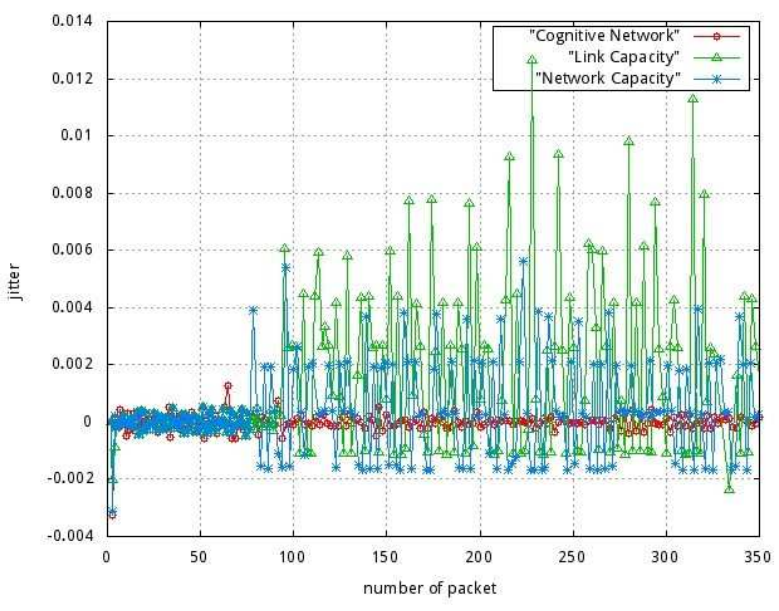

Fig. 5: Average jitter

\subsection{Session completion rate of the mobile users}

In this scenario, we consider the case session completion rate of the mobile users, and simulation time is $300 \mathrm{~s}$. We simulated a rectangular area of $600 \times 300$ with two Access Points placed on either side of the center position, and 60 randomly placed users. The maximum number of connections for each access point is set to 30. CBR data sources are set at a rate of 0.25 packets per second. Individual nodes can randomly move in any direction, the pause time is set to 10s. The results are shown in Fig. 6. The network capacity scenarios, when the numbers of nodes are more than 40, the network session completion rate sharply declined. The Cognitive Network Session has a better performance for Quantum Genetic Algorithm .The result is displayed in Fig. 7. As the nodes accelerate their movement the end-to-end packet delay of two access schemes have increased, but cognitive network delay increases more slowly and the delay stabilizes after achieving a speed of around $25 \mathrm{~m} / \mathrm{s}$. For the link capacity access scheme, as the speed of the nodes increases, the network delays also keeps increasing. The results show that cognitive network access scheme has a more superior performance result compared to the other two schemes.

\subsection{Network capacity to respond to sudden data burst}

To quantify the control traffic overhead for the Cognitive scheme, we considered a simple implementation with burst traffic. Since VoIP requires low delay, video needs to higher bandwidth, and file transfer demand on higher reliability. To evaluate this scenario, we set 11 user nodes, of which 10 use Pareto on/off flow model. Every ten seconds a user requests access to the $\mathrm{AP}$ and one node uses the variable bit rate (VBR) traffic generated for the 


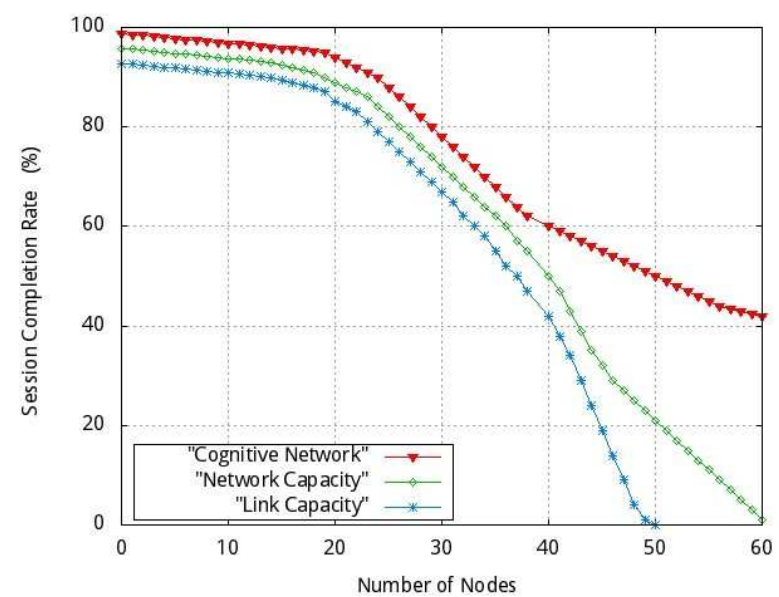

Fig. 6: Session completion rate

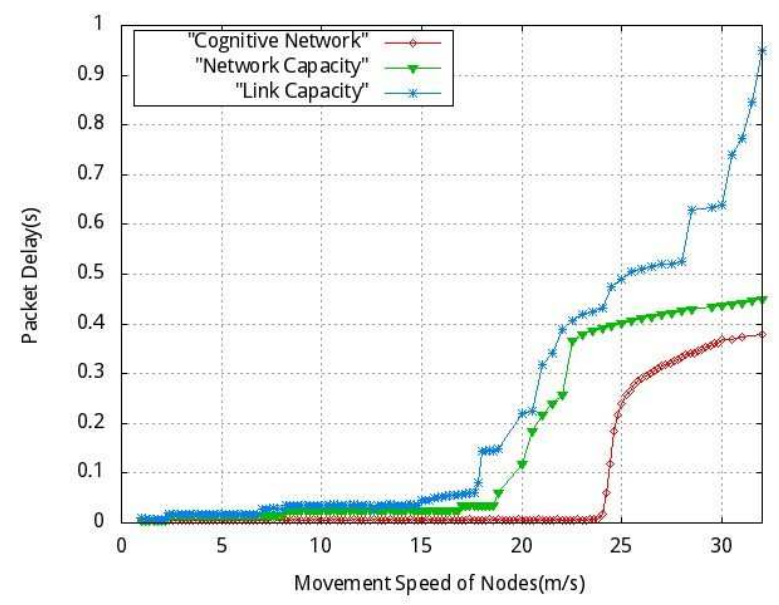

Fig. 7: Average delay

simulation of large data traffic by a network user. The parameters used in this scenario are the same as used for simulation in section 4.1. Simulation time is $400 \mathrm{~s}$.

As shown in Fig. 8, during the first $100 \mathrm{~s}$ of the simulation, the number of active users accessing the two APs have gradually increased. Four users accessed AP1 and six users accessed AP2. At time $110 \mathrm{~s}$, when the VBR traffic user joins AP1, the performance of AP1 starts declining. The result is that much more recourses become available to users that are connected to AP2. So, in Cognitive Network access methods, one of the users connected to AP1 joins AP2, which improves the overall network performance. At time $210 \mathrm{~s}$, the VBR user has finished transmitting data left AP1. A portion of the users connected to AP2 has joined AP1. As shown in Fig. 9, the performance of the cognitive network method is always better than the network capacity method as it has a low end-to-end packet loss rate.

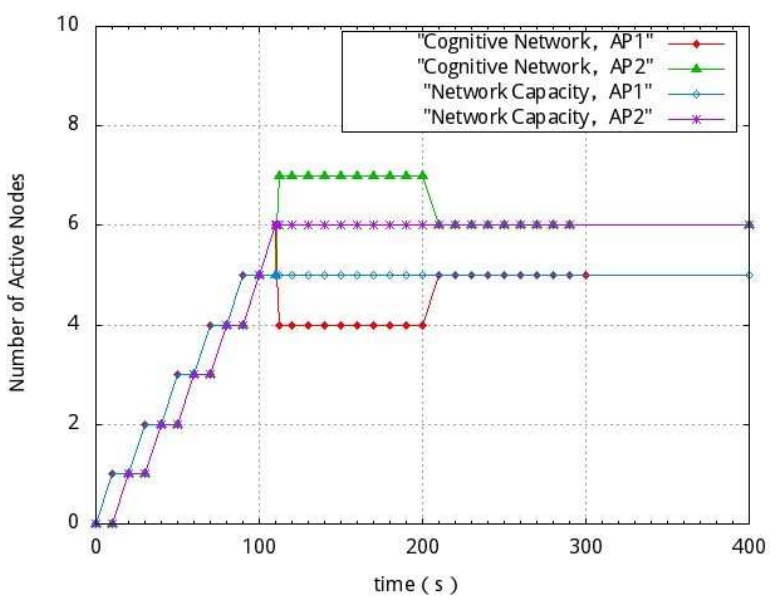

Fig. 8: Number of active nodes

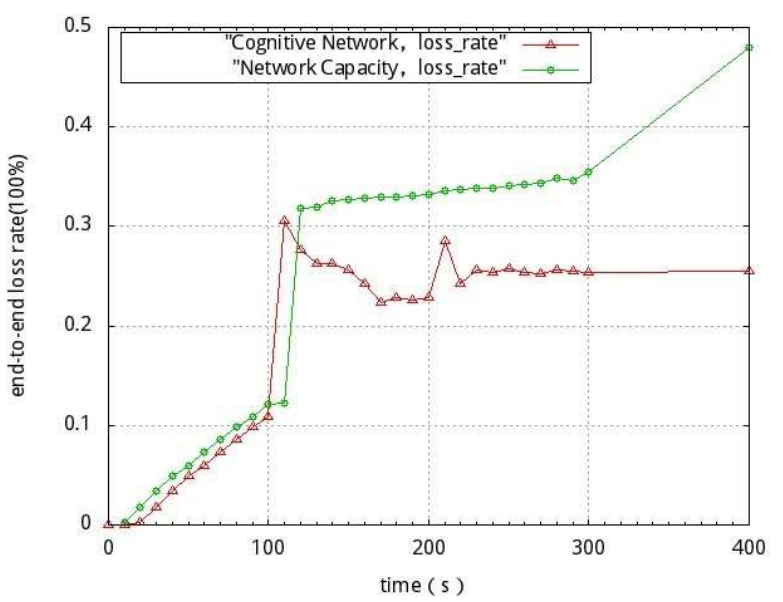

Fig. 9: End-to-end loss rate

\section{Conclusion}

In this paper we have proposed a cognitive network access method in which the network performance reported by all previous users is compared to the requirements of the current users. The expected end-to-end link performance of the current users is considered by our system in order to achieve the best access opportunity. The framework features a modular design and a generic knowledge representation based on fuzzy comprehensive evaluation method, which together with quantum genetic algorithm proves to achieve better performance.

The results have shown that the cognitive network access scheme proposed in this paper performs significantly better than the other state-of-the art schemes, in terms of both overall and end-to-end performance. Some interesting directions for future research include a 
detailed analysis of the extent to which the use of fuzzy comprehensive evaluation in handling the imprecision and uncertainty of the performance characterization of wireless networks. Therefore, in future, we intend to focus our research to add more access technologies such as WiMAX, GPS and so on. This will give the users more options to selected to a much wider pool of access networks and hence a greater chance of improving the overall network performance.

\section{Acknowledgements}

The work described in this paper is based on the results of the National Natural Science Foundation of China (60973027).

Part of this work was supported by grant from the National High Technology Research and Development Program of China (2007AA01Z401), the Fundamental Research Fund for the Central Universities (HEUCF100601, HEUCFZ1213), the Research Fund for the Doctoral Program of Higher Education of China (20102304120012), the Natural Science Foundation of Heilongjiang Province (ZD201102, F201037), the Research Fund for Scientific and Technological Innovation of Harbin (RC2011XK010004, 2011RFQXG035, 2011AA1CG005, RC2011QN01 0019), and the Postdoctoral Science Foundation of Heilongjiang Province(LBH-210204).

\section{References}

[1] R. Wei, A. Swami Z. Qing. Connectivity of Heterogeneous Wireless Networks. IEEE Transactions on Information Theory, 57, 4315-4331 (2011).

[2] Q. Mahmud. Cognitive Networks: Towards Self-aware Networks. NY, Wiley-Interscience, 1-59 (2007).

[3] C. Marius, F. Jens, et al. Access Network Discovery and Selection in the Future Wireless Communication. Mobile Networks and Applications. Mag., 16, 337-349 (2011).

[4] G. Song. J. Wu ,J. Schormans, et al. A Performance Study of Hierarchical Heterogeneous Wireless Integrated Networks. Applied Mathematics \& Information Sciences. Mag., 6, 497504 (2012).

[5] A. J. Hartwell, J. R. Davies, et al. A sensor network cross-layer power control algorithm that incorporates multiple-access interference. IEEE Transactions on Wireless Communications, 7, 2877-2883 (2008).

[6] V. Srivastava, M. Magazine. Cross-Layer Design: A Survey and the Road Ahead, CROSS-LAYER DESIGN. Mag., 43, $112-119$ (2005).

[7] F. Carolina, M. Mihael. Trends in the development of communication networks: Cognitive networks. Computer Network. Mag., 53, 1354-1376 (2009).

[8] R. W. Thomas, L. A. DaSilva, A. B. MacKenzie Cognitive networks. 2005 IEEE International Symposium on New Frontiers in Dynamic Spectrum Access Networks, 2, 352-360 (2005).
[9] C. Mung, S.H. Low, et al. Layering as optimization decomposition: A mathematical theory of network architectures. Proceedings of the IEEE, 95, 255-312 (2007).

[10] Z. Guan, T. Melodia, et al. Distributed spectrum management and relay selection in interference-limited cooperative wireless networks. Proceedings of the 17th annual international conference on Mobile computing and networking, 229-240 (2012).

[11] W. Shi, S. Fan, et al. Fuzzy neural network based access selection algorithm in heterogeneous wireless networks. Tongxin Xuebao/Journal on Communications. Mag., 31, 151156 (2011).

[12] S. Navarro, L. Yuxia, et al.. An MDP-based vertical handoff decision algorithm for heterogeneous wireless networks. IEEE Transactions on Vehicular Technology, 57, 1243-1254 (2008).

[13] S. A. Sharna, M. R. Amin, M. Murshed. An EnhancedMDP Based Vertical Handoff Algorithm for QoS Support over Heterogeneous Wireless Networks. Proceedings 2011 IEEE International Symposium on Network Computing and Applications, 289-293 (2011).

[14] R. Ketan,G. Nikolaos, et al. Cross-layer design of coded multicast for wireless random access networks. Proceedings of 2011 45th Annual Conference on Information Sciences and Systems, 29, 1-6 (2011).

[15] S.U. Khan, I. Ahmad. A pure nash equilibrium-based game theoretical method for data replication across multiple servers. IEEE Transactions on Knowledge and Data Engineering. Mag., 21, 537-553 (2009)

[16] V. Robert, J.K. George, et al. Fuzzy Logic in Geology, 121151 (2004).

[17] H. Xing, X. Liu, et al. A quantum-inspired genetic algorithm for k-means clustering. Computer Communications. Mag., 32, 386-393 (2009).

[18] http://www.isi.edu/nsnam/ns/, (2012). 


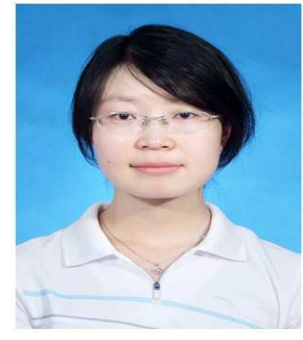

Xiaoming Chen received the MS degree in Computer Application Technology from the Department of Computer Science and Technology, Harbin Engineering University in 2010. She is currently a doctoral candidate in Harbin Engineering University. Her research interests are in the areas of cognitive networks, cross-layer design and network architecture.

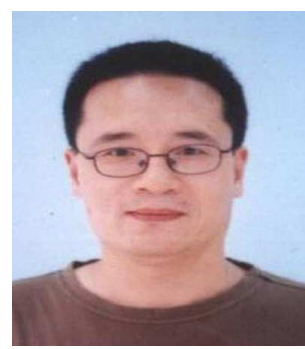

Huiqiang Wang received the MS degree in information and computing science from Harbin Engineering University in 1985, and the $\mathrm{PhD}$ degree in computer application technology from the department of Computer science and technology, Harbin Engineering University in 2005. He is currently a professor in Harbin Engineering University. His research interests are in the areas of network technology and information security, trusted computing, autonomic computing, cognitive networks.

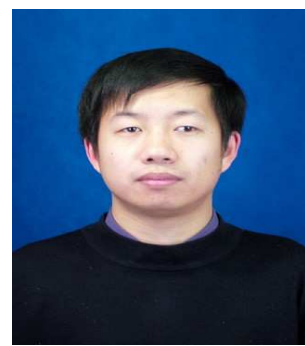

Hongwu Lv received the MS degree in information and computing science from Harbin Engineering University in 2006, and the $\mathrm{PhD}$ degree in computer application technology from the department of Computer science and technology, Harbin Engineering University in 2011. He is currently a lecture in Harbin Engineering University. His research interests are in the areas of performance evaluation and autonomic computing.

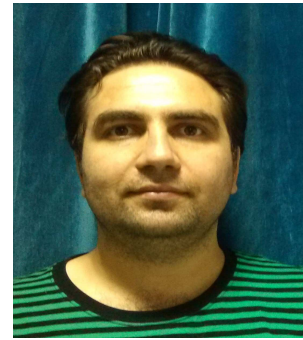

Amjad Ali is a $\mathrm{PhD}$ student at Harbin Engineering University. $\mathrm{He}$ received his BS degree in computer science from University of Peshawar in 2001. He received his MS degree in computer science from Agricultural University Peshawar in 2004. He has also received MS degree in computer based communication networks from Institute of Management Sciences, Hayatabad in 2008. He is the author of several research papers. His research interests include routing in mobile ad hoc networks, cognitive networks and multi-channel MAC protocols for wireless networks.

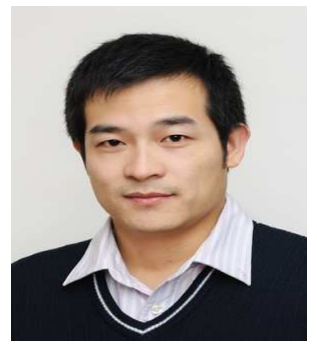

Zhendong Wang received his B.S. and M.S. degrees in Information \& Computing Sciences and Technology of Computer Application from Changchun University of Science and Technology and Harbin University of Science and Technology in 2006 and 2009 respectively. $\mathrm{He}$ is recently working towards his Ph.D. degrees at Harbin Engineering University, Harbin, China. His research interests span the broad area of cognitive radio networks spectrum sensing and access. 